\title{
Converence and submeasures in Boolean algebras
}

\author{
Thomas Jech \\ e-mail: jech@math.psu.edu
}

October 8, 2018

In memory of Bohuslav Balcar

\begin{abstract}
A Boolean algebra carries a strictly positive exhaustive submeasure if and only if it has a sequential topology that is uniformly Fréchet.
\end{abstract}

\section{Introduction.}

In 1937, John von Neumann proposed the problem of characterizing the complete Boolean algebras that carry a strictly positive $\sigma$-additive measure (The Scottish Book [17, Problem 163.) Such algebras satisfy the countable chain condition (ccc): every subset of mutually disjoint elements is countable. In addition, von Neumann isolated a weak distributive law and asked whether these two properties are sufficient for the existence of measure.

The first major contribution to von Neumann's problem was given by Dorothy Maharam [16] who identified a part of the problem as a topological problem. She showed that a complete Boolean algebra carries a strictly positive continuous submeasure if and only if the sequential topology given by the algebraic convergence is metrizable. This gives a natural decomposition of the von Neumann problem into two parts:

I. Does every complete weakly distributive ccc Boolean algebra carry a strictly positive continuous submeasure? 
II. Does the existence of a strictly positive continuous submeasure imply the existence of a strictly positive $\sigma$-additive measure?

Part II has become the well known and well-studied Control Measure Problem [12] in Functional Analysis and remained open until Talagrand's solution [22] in 2008.

As for Part I, Maharam showed that a negative answer would follow from the existence of a Suslin algebra. Its existence is equivalent to the negation of Suslin's Hypothesis [20] from 1920 but was not known until 1967 (cf. [23], 9].) The existence of a Suslin algebra is not provable; it is only consistent with the axioms of set theory [19]. The work of Balcar, Jech and Pazák [3] in 2003 established that the positive answer Part I of Maharam's problem is also consistent.

The work on Maharam's problem I followed in Maharam's footsteps and was carried out mostly by Bohuslav Balcar and his collaborators (cf. [1], 2], 3], 4], [5]), with additional contributions of Stevo Todorcevic 24, 25]. The metrizability assumption of Maharam was successively weakened to "ccc and Hausdorff" [1], "ccc, weakly distributive and $G_{\delta}$ " [3], "weakly distributive and $\sigma$-finite cc" [25], and "uniformly weakly distributive" [4].

In this paper we generalize the topological characterization to finitely additive measures on Boolean algebras, when neither the algebra nor the measure are complete. As the Control Measure Problem has been solved by Talagrand, and there exists an exhaustive submeasure that is not equivalent to a measure, the goal is to characterize Boolean algebras that carry an exhaustive submeasure. We give a necessary and sufficient condition in Theorem 3.6.

Our investigations were inspired by the work of Bohuslav Balcar who advocated the use of sequential topology and who introduced me to Maharam's problems. This paper is dedicated to his memory.

\section{Submeasures on Boolean algebras}

A Boolean algebra is a set $B$ with Boolean operations $a \vee b$ (join), $a \wedge b$ (meet), $-a$ (complement) and $a \triangle b$ (symmetric difference), the partial order $a \leq b$, and the smallest and greatest element, $\mathbf{0}$ and $\mathbf{1}$. To avoid trivialities we assume that $B$ is infinite and atomless. We let $B^{+}=B-\{\mathbf{0}\}$.

An antichain in $B$ is a set $A \subset B$ whose any two elements $a, b$ are disjoint i.e. $a \wedge b=\mathbf{0} . B$ satisfies the countable chain condition (ccc) 
if it has no uncountable antichains.

Definition 2.1. A submeasure on a Boolean algebra $B$ is a real valued function $m$ on $B$ such that

(i) $m(\mathbf{0})=0$ and $m(\mathbf{1})=1$,

(ii) if $a \leq b$ then $m(a) \leq m(b)$,

(iii) $m(a \vee b) \leq m(a)+m(b)$.

A submeasure $m$ is strictly positive if

(iv) $m(a)=0$ only if $a=\mathbf{0}$.

$m$ is a measure if

(v) $m(a \vee b)=m(a)+m(b)$ whenever $a$ and $b$ are disjoint.

Following Talagrand [21], a submeasure $m$ is exhaustive if for every infinite antichain $\left\{a_{n}\right\}_{n}, \lim _{n} m\left(a_{n}\right)=0 ; m$ is uniformly exhaustive if for every $\varepsilon>0$ there exists a $K$ such that every antichain has at most $K$ elements with $m(a) \geq \varepsilon$.

Note that some condition like "exhaustive" is needed to exclude trivial examples of submeasures like $m(a)=1$ for all $a \neq 0$. Every measure is uniformly exhaustive, and uniformly exhaustive implies exhaustive.

If $m$ a strictly positive exhaustive submeasure, let, for each $n \in \mathbf{N}$,

$$
C_{n}=\{a \in B: m(a) \geq 1 / n\} .
$$

Then $C_{1} \subset C_{2} \subset \ldots \subset C_{n} \subset \ldots$ and $\bigcup_{n} C_{n}=B^{+}$, and for each $n$, every antichain in $C_{n}$ is finite. If $m$ is uniformly exhaustive then there exist $K_{n}, n \in \mathbf{N}$, such that for each $n$, every antichain in $C_{n}$ has size at most $K_{n}$. We say that $B$ is $\sigma$-finite $c c$, resp. $\sigma$-bounded $c c$.

In [13, Kalton and Roberts proved that if $m$ is a uniformly exhaustive submeasure then it is equivalent to a measure. This reduced the Control Measure Problem to the question whether ther exists an exhaustive submeasure that is not uniformly exhaustive. Such a submeasure was constructed by Talagrand in [22].

Let $m$ be a strictly positive exhaustive submeasure. Letting

$$
\rho(a, b)=m(a \triangle b),
$$


we obtain a distance function that makes $B$ a metric space. The metric $\rho$ defines a topology on $B$ that is invariant under Boolean operations; in particular if $U$ is an open neighborhood of $\mathbf{0}$ then $a \Delta U$ is an open neighborhood of $a$.

We shall isolate a property of topological Boolean algebras that is necessary and sufficient for the existence of an exhaustive submeasure.

\section{Convergence ideals on Boolean al- gebras}

We consider an abstract theory of convergent sequences in topological Boolean algebras. This is inspired by an earlier work of Balcar, e.g. [1] and [2].

Definition 3.1. Let $B$ be a Boolean algebra. $A$ convergence ideal on $B$ is a set $I$ of infinite sequences $\left\{a_{n}\right\}_{n}$ in $B$ with the following properties:

(i) $\bigwedge_{n} a_{n}=\mathbf{0}$, i.e. there is no $a>\mathbf{0}$ such that $a \leq a_{n}$ for all $n$.

(ii) If $s \in I$ and if $t$ is an infinite subsequence of $s$ then $t \in I$.

(iii) If $\left\{a_{n}\right\}_{n} \in I$ and $b_{n} \leq a_{n}$ for all $n$ then $\left\{b_{n}\right\}_{n} \in I$.

(iv) If $\left\{a_{n}\right\}_{n} \in I$ and $\left\{b_{n}\right\}_{n} \in I$ then $\left\{a_{n} \vee b_{n}\right\}_{n} \in I$.

(v) If $\left\{a_{n}\right\}_{n}$ is an infinite antichain then $\left\{a_{n}\right\}_{n} \in I$.

Given a convergence ideal $I$ we write $\lim _{n} a_{n}=\mathbf{0}$ instead of $\left\{a_{n}\right\}_{n} \in$ $I$ and say that $\left\{a_{n}\right\}$ converges to $\mathbf{0}$. We extend this to convergence to any $a$ by

$$
\lim a_{n}=a \text { whenever } \lim \left(a_{n} \Delta a\right)=\mathbf{0} .
$$

This notion of convergence has the obvious properties like

-each $a_{n}$ converges to at most one limit,

-each constant sequence converges to the constant

$-\lim \left(a_{n} \vee b_{n}\right)=\lim a_{n} \vee \lim b_{n}$, etc.

Given a convergence ideal we define a topology on $B$ as follows: a set $A$ is closed if it contains the limits of all sequences in $A$. We call such a topology sequential. 
Definition 3.2. A sequential topology on $B$ is Fréchet if for every $A$, the closure of $A$ is the set of all limits of sequences in $A$.

It should be clear that a sequential topology is Fréchet if and only if it has this property:

For every $A$, if for every $k \in \mathbf{N},\left\{a_{n}^{k}\right\}_{n}$ is a sequence in $A$ with $\lim _{n} a_{n}^{k}=b_{k}$, and if $\lim _{k} b_{k}=c$, then there exists a sequence $\left\{c_{k}\right\}_{k}$ in $A$ such that $\lim _{k} c_{k}=c$.

Lemma 3.3. A sequential topology on $B$ is Fréchet if and only if the convergence ideal has the diagonal property:

if $\lim _{n} x_{n}^{k}=\mathbf{0}$ for all $k$, then there exists a function $F: \mathbf{N} \rightarrow \mathbf{N}$ such that $\lim _{k} x_{F(k)}^{k}=\mathbf{0}$.

Proof. Assume that $I$ has the diagonal property and let $a_{n}^{k}, b_{k}$ and $c$ be such that $\lim _{n} a_{n}^{k}=b_{k}$ and $\lim _{k} b_{k}=c$. We shall find a function $F: \mathbf{N} \rightarrow \mathbf{N}$ such that $\lim _{k} a_{F(k)}^{k}=c$.

Let $x_{n}^{k}=a_{n}^{k} \Delta b_{k}$; then $\lim _{n} x_{n}^{k}=\mathbf{0}$ for each $k$, and so there exists a function $F$ such that $\lim _{k} x_{F(k)}^{k}=\mathbf{0}$. Now because

$$
a_{F(k)}^{k} \Delta c \leq\left(a_{F(k)}^{k} \Delta b_{k}\right) \vee\left(b_{k} \Delta c\right),
$$

we have

$$
\lim _{k}\left(a_{F(k)}^{k} \Delta c\right) \leq \lim _{k}\left(a_{F(k)}^{k} \Delta b_{k}\right) \vee \lim _{k}\left(b_{k} \Delta c\right)=\mathbf{0}
$$

and so $\lim _{k} a_{F(k)}^{k}=c$.

For the converse, assume that the sequential topology is Fréchet and let $x_{n}^{k}$ be such that $\lim _{n} x_{n}^{k}=\mathbf{0}$ for each $k$. Let $A=\left\{a_{k}\right\}_{k}$ be an infinite antichain, and let, for each $n$,

$$
y_{n}^{k}=x_{n}^{0} \vee x_{n}^{1} \vee \ldots \vee x_{n}^{k}, \text { and } z_{n}^{k}=y_{n}^{k} \vee a_{k} .
$$

For each $k$ we have $\lim _{n} y_{n}^{k}=\mathbf{0}$ and so $\lim _{n} z_{n}^{k}=a_{k}$.

Let $Z=\left\{z_{n}^{k}: k, n \in \mathbf{N}\right\}$. Each $a_{k}$ is in the closure of $Z$, and so is $\mathbf{0}$ since $\mathbf{0}=\lim _{k} a_{k}$. Because the topology is Fréchet, there is a sequence in $Z$ with limit $\mathbf{0}$; hence there is an infinite set $E \subset \mathbf{N} \times \mathbf{N}$ such that $\lim \left\{z_{n}^{k}:(k, n) \in E\right\}=\mathbf{0}$. For each $k$ there are only finitely many $n$ with $(k, n) \in E$; this is because $\lim _{n} z_{n}^{k}=a_{k}$. Hence there is an infinite subset $M$ of $\mathbf{N}$ and a function $G$ on $M$ such that $\lim \left\{z_{G(m)}^{m}: m \in\right.$ $M\}=\mathbf{0}$. Let $M=\{m(0)<m(1)<\ldots<m(k)<\ldots: k \in \mathbf{N}\}$ and let $F(k)=G(m(k))$. Then we have, for every $k$,

$$
x_{F(k)}^{k} \leq y_{F(k)}^{k} \leq y_{F(k)}^{m(k)} \leq z_{G(m(k))}^{m(k)}
$$


and so $\lim _{k} x_{F}^{k}(k)=\mathbf{0}$.

Definition 3.4. The sequential topology given by a convergence ideal $I$ is uniformly Fréchet if there exist choice functions $F_{k}$ acting on infinite sequences such that, whenever $\left\{x_{n}^{k}\right\}_{n}, k \in \mathbf{N}$, are sequences converging to $\mathbf{0}$, then

$$
\lim _{k} F_{k}\left(\left\{x_{n}^{k}\right\}_{n}\right)=\mathbf{0}
$$

(the kth term is the term of the kth sequence chosen by the kth function.)

This property is the uniform version of the diagonal property: the element chosen from the $k$ th sequence depends only on $k$ and the sequence, not on the other sequences.

Proposition 3.5. Let $m$ be a strictly positive exhaustive submeasure on $B$, and let $I$ be the ideal of all sequences $\left\{a_{n}\right\}_{n}$ in $B$ such that $\lim _{n} m\left(a_{n}\right)=0$. Then $I$ is a convergence ideal and the topology is uniformly Fréchet.

Proof. For $\left\{a_{n}\right\}_{n} \in I, F_{k}$ chooses some $a_{n}$ such that $m\left(a_{n}\right)<1 / k$.

Theorem 3.6. A Boolean algebra B carries a strictly positive exhaustive submeasure if and only if it has a uniformly Fréchet convergence ideal.

We prove the theorem in the next Section.

\section{$4 \quad$ Fragmentations}

If $m$ is a measure (or an exhaustive submeasure) on a Boolean algebra $B$, let for each $n$

$$
C_{n}=\{a: m(a) \geq 1 / n\} .
$$

Every antichain in every $C_{n}$ is finite (or of bounded size if $m$ is uniformly exhaustive) and so (in modern terminology), $B$ is $\sigma$-finite cc, or $\sigma$-bounded cc. These properties were considered in [8] and [7]. More recently, approximations of $B$ by a chain $\left\{C_{n}\right\}_{n}$ of sets with various properties have proved useful, see e.g. [4], p. 260. In Balcar's terminology, such approximations are called fragmentations. 
Definition 4.1. $A$ fragmentation of a Boolean algebra $B$ is a sequence of subsets $C_{1} \subset C_{2} \subset \ldots \subset C_{n} \subset \ldots$ such that $\bigcup_{n} C_{n}=B^{+}$and for every $n$, if $a \in C_{n}$ and $a \leq b$ then $b \in C_{n}$.

$A$ fragmentation is $\sigma$-finite cc if for every $n$, every antichain in $C_{n}$ is finite.

A fragmentation is $\sigma$-bounded cc if for every $n$ there is a constant $K_{n}$ such that every antichain $A \subset C_{n}$ has size $\leq K_{n}$.

$A$ fragmentation is graded if for every $n$, whenever $a \cup b \in C_{n}$ then either $a \in C_{n+1}$ or $b \in C_{n+1}$.

In this Section we prove that if $B$ has a uniformly Fréchet ideal $I$ then $B$ has a graded $\sigma$-finite cc fragmentation.

Let $I$ be a uniformly Fréchet ideal and let $F_{k}, k \in \mathbf{N}$, be choice functions from definition 3.4. For each $n$, let

$V_{n}=\left\{a: a \leq F_{n}(\vec{x})\right.$ for some $\left.\vec{x} \in I\right\}, U_{n}=V_{1} \cap \ldots \cap V_{n}, C_{n}=B-U_{n}$.

We claim that $\left\{C_{n}\right\}_{n}$ is a fragmentation: it is enough to show that each $a \neq \mathbf{0}$ is in some $C_{n}$. If $a \notin C_{n}$ for all $n$, then for every $n$ there exists some $\vec{x}^{n} \in I$ such that $a \leq F_{n}\left(\vec{x}^{n}\right)$. By the uniform diagonality, $\left\{F_{n}\left(\vec{x}^{n}\right)\right\}_{n} \in I$ and it follows that $a=\mathbf{0}$.

A similar argument shows

Lemma 4.2. If $\left\{a_{n}\right\}_{n}$ is such that $a_{n} \notin C_{n}$ for each $n$ then $\left\{a_{n}\right\}_{n} \in I$.

Proof. For each $n$ there exists some $\vec{x}^{n} \in I$ such that $a_{n} \leq F_{n}\left(\vec{x}^{n}\right)$ Because $\left\{F_{n}\left(\vec{x}^{n}\right)\right\}_{n} \in I$, we have $\left\{a_{n}\right\}_{n} \in I$.

Lemma 4.3. For every $n$, no $\vec{a} \in I$ is a subset of $C_{n}$.

Proof. If some $\vec{a} \in I$ is a subset of $C_{n}$ then for some $k \leq n$, some infinite subsequence $\vec{b}$ of $\vec{a}$ is disjoint from $V_{k}$. But $F_{k}(\vec{b}) \in V_{k}$.

Note that the fragmentation is $\sigma$-finite cc, because every infinite antichain is in $I$.

Lemma 4.4. For every $n$ there exists a $k$ such that $U_{k} \vee U_{k} \subset U_{n}$. Hence $\left\{C_{n}\right\}_{n}$ has a subfragmentation that is $\sigma$-finite cc and graded.

Proof. Let $n \in \mathbf{N}$ and assume that for each $k$ there exist $a_{k}$ and $b_{k}$ in $U_{k}$ such that $c_{k}=a_{k} \vee b_{k} \in C_{n}$. By Lemma 4.2, $\left\{a_{k}\right\}_{k}$ and $\left\{b_{k}\right\}_{k}$ are in $I$ and so $\left\{c_{k}\right\}_{k} \in I$. But $\left\{c_{k}\right\}_{k} \subset C_{n}$, a contradiction. 


\section{Construction of an exhaustive sub- measure}

let $B$ be a Boolean algebra and assume that $B$ has a graded $\sigma$-finite cc fragmentation $\left\{C_{n}\right\}_{n}$. We shall define a submeasure on $B$.

For each $n$ let $U_{n}=B-C_{n}$ and $U_{0}=B$; we have $U_{0} \supset U_{1} \supset \ldots \supset$ $U_{n} \supset \ldots$ and $\bigcap_{n} U_{n}=\{\mathbf{0}\}$. For $X, Y \subset B$, let $X \vee Y$ denote the set $\{x \cup y: x \in X, y \in Y\}$. As the fragmentation is graded, we have

$$
U_{n+1} \vee U_{n+1} \subset U_{n}
$$

for all $n \geq 0$. Cosequently, if $n_{1}<\ldots<n_{k}$ then

$$
U_{n_{1}+1} \vee \ldots \vee U_{n_{k}+1} \subset U_{n_{1}}
$$

This is proved by induction on $k$.

Let $D$ be the set of all $r=\sum_{i=1}^{k} 1 / 2^{n_{i}}$ where $0<n_{1}<\ldots<n_{k}$. For each $r \in D$ we let $V_{r}=U_{n_{1}} \vee \ldots \vee U_{n_{k}}$ and $V_{1}=U_{0}=B$. For each $a \in B$ define

$$
m(a)=\inf \left\{r \in D \cup\{1\}: a \in V_{r}\right\} .
$$

Using the above property of the $U_{n}$, it follows that $V_{r} \subset V_{s}$ if $r<s$, and $V_{r} \vee V_{s} \subset V_{r+s}$ when $r+s \leq 1$. From this we have $m(a) \leq m(b)$ if $a \subset b$, and $m(a \cup b) \leq m(a)+m(b)$.

The submeasure $m$ is strictly positive because if $a \neq \mathbf{0}$ then for some $n, a \notin U_{n}$, and hence $m(a) \geq 1 / 2^{n}$.

Finally, $m$ is exhaustive: If $A$ is an infinite antichain, then for each $n$ only finitely many elements of $A$ belong to $C_{n}$ and hence only finitely many have $m(a) \geq 1 / 2^{n}$.

\section{Measures and submeasures}

Following Maharam's work [16], the early work on the von Neumann Problem and the Control Measure Problem isolated the following classes of Boolean algebras: complete Boolean algebras that carry a $\sigma$-additive measure (measure algebars), complete Boolean algebras that carry a continuous submeasure (Maharam algebras), Boolean algebras that carry a finitely additive measure and Boolean algebras that carry exhaustive submeasure. (For a detailed analysis, see Fremlin's article [6] in the Handbook of Boolean Algebras [18], and Balcar et al. [1].) 
The Control Measure Problem was reduced to the question whether every exhaustive submeasure is equivalent to a finitely additive measure. The result of Kalton and Roberts [13] shows that every uniformly exhaustive submeasure is equivalent to a finitely additive measure, and in 2007, Michel Talagrand answered the question in [22] by constructing an exhaustive measure (on a countable Boolean algebra) that is not uniformly exhaustive.

Returning to the characterization of algebras with submeasure by convergence, we have the following:

Definition 6.1. Let $I$ be a convergence ideal on a Boolean algebra $B$. $B$ is $I$ - concentrated if for every sequence $\left\{A_{n}\right\}_{n}$ of finite antichains with $\left|A_{n}\right| \geq n$ there exists a sequence $\left\{a_{n}\right\}_{n} \in I$ such that $a_{n} \in A_{n}$ for all $n$.

Theorem 6.2. A Boolean algebra carries a strictly positive finitely additive measure if and only if it has a uniformly Fréchet convergence ideal $I$ and is I-concentrated.

Proof. Let $\left\{C_{n}\right\}_{n}$ be the fragmentation constructed in Section 4 , We claim that each $C_{k}$ has a bound on the size of antichains Otherwise, let $A_{n}$ be antichains in $C_{k}$ of size at least $n$, and let $\left\{a_{n}\right\}_{n} \in I$ be such that $a_{n} \in A_{n}$. This contradicts Lemma 4.3.

It follows that $B$ has a $\sigma$-bounded cc graded fragmentation. Using the Kalton-Roberts method, one can verify Kelley's condition [15] for the existence of a strictly positive measure; for details, see [11].

We wish to mention a subtle point here. In the proof of Theorem 6.2 we use a graded $\sigma$-bounded cc fragmentation. It is not enough to have a $\sigma$-bounded cc algebra with a graded fragmentation: the metric completion of Talagrand's example is a Maharam algebra that does not carry a measure but is $\sigma$-bounded cc.

\section{Continuity and weak distributivity}

Let $B$ be a complete Boolean algebra. $B$ has a $\sigma$-additive measure if and only if it has finitely additive measure and is weakly distributive. This is explicitly stated in Kelley's [15] but was already known to Pinsker, see [14]. Moreover, the proof of continuity is exactly the same when applied to submeasures, see Fremlin [6], p. 946: 
Theorem 7.1. Let $B$ be a complete Boolean algebra.

(a) $B$ carries a strictly positive $\sigma$-additive measure if and only if it is weakly distributive and carries a strictly positive finitely additive measure.

(b) $B$ carries a strictly positive continuous submeasure if and only if it is weakly distributive and carries a strictly positive exhaustive sumbeasure.

We note that (for ccc algebras) weak distributivity is equivalent to the Fréchet property for the algebraic convergence (and uniform weak distributivity is equivalent uniformly Fréchet.) Thus:

Theorem 7.2. Let $B$ be a complete Boolean algebra.

(a) $B$ is a Maharam algebra if and only if it is uniformly weakly distributive [4], [5].

(b) $B$ is a measure algebra if and only if it is uniformly wekaly distributive and concentrated [10].

\section{Fréchet and uniformly Fréchet}

The Fréchet property does not (provably) imply uniform Fréchet. This is because a Suslin algebra (the algebra obtained from a Suslin tree) is weakly distributive but not $\sigma$-finite cc. This counterexample is only consistent with ZFC ([23], [9]) and does not exist in some models of set theory [19]. Moreover, by [3] it is consistent that every weakly distributive ccc complete Boolean algebra is a Maharam algebra (and hence uniformly weakly distributive). This shows that Fréchet could imply uniformly Fréchet for the algebraic convergence but the proof does not seem to work in general. Thus we end with the following open problem:

Problem 1. Is there (in ZFC) a Boolean algebra with a convergence ideal I that is Fréchet but not uniformly Fréchet?

\section{References}

[1] B. Balcar, W. Główczyński, and T. Jech. The sequential topology on complete Boolean algebras. Fund. Math., 155(1):59-78, 1998. 
[2] B. Balcar, F. Franěk, J. Hruška. Exhaustive zero-convergence structures on Boolean algebras. Acata Univ. Carolinae, Math. et Phys., 40: 27-41, 1999.

[3] B. Balcar, T. Jech, and T. Pazák. Complete ccc Boolean algebras, the order sequential topology and a problem of von Neumann. Bull. London Math. Society, 37:885-898, 2005.

[4] Bohuslav Balcar and Thomas Jech. Weak distributivity, a problem of von Neumann and the mystery of measurability. Bull. Symbolic Logic, 12(2):241-266, 2006.

[5] B. Balcar and T. Jech. Contributions to the theory of weakly distributive complete Boolean algebras. A Tribute to Andrzej Mostowski, A. Ehrenfeucht et al., eds., 2007

[6] D. H. Fremlin. Measure algebras. In Handbook of Boolean algebras. Vol. 3, pages 877-980. North-Holland Publishing Co., Amsterdam, 1989.

[7] H. Gaifman. Concerning measures on Boolean algebras. Pacific J. Math., 14: 61-73, 1964.

[8] A. Horn and A. Tarski. Measures in Boolean algebras. Trans. Amer. Math. Soc., 64:467-497, 1948.

[9] T. Jech. Non-provability of Souslin's hypothesis. Comment. Math. Univ. Carolinae, 8:291-305, 1967.

[10] T. Jech. Algebraic characterizations of measure algebras. Proc. Amer. Math. Soc., 136:1285-1294, 2008.

[11] T. Jech. Measures on Boolean algebras. Fundamenta Math, to appear

[12] N. J. Kalton. The Maharam problem. Publ. Math. Univ. Pierre et Marie Curie 94, Univ. Paris VI, Paris, 1989.

[13] N. J. Kalton and J. W. Roberts. Uniformly exhaustive submeasures and nearly additive set functions. Trans. Amer. Math. Soc., 278:803-816, 1983.

[14] L. V. Kantorovič, B. Z. Vulikh, and A. G. Pinsker. Functional Analysis in Partially Ordered Spaces. 1950. (in Russian). 
[15] J. L. Kelley. Measures on Boolean algebras. Pacific J. Math., 9:1165-1177, 1959.

[16] D. Maharam. An algebraic characterization of measure algebras. Ann. of Math. (2), 48:154-167, 1947.

[17] D. Mauldin, editor. The Scottish Book. Birkhäuser Boston, Mass., 1981.

[18] J. D. Monk, editor. Handbook of Boolean algebras. North-Holland Publishing Co., Amsterdam, 1989.

[19] R. M. Solovay and S. Tennenbaum. Iterated Cohen extensions and Souslin's problem. Ann. of Math. (2), 94:201-245, 1971.

[20] M. Suslin. Problème 3. Fund. Math., 1:223, 1920.

[21] M. Talagrand. A simple example of pathological submeasure. Math. Ann., 252:97-102, 1979/80.

[22] M. Talagrand Maharam's Problem. Ann. of Math. (2), 168:9811009, 2008.

[23] S. Tennenbaum. Souslin's problem. Proc. Nat. Acad. Sci. U.S.A., 59:60-63, 1968.

[24] S. Todorcevic. A dichotomy for P-ideals of countable sets. Fund. Math., 166(3):251-267, 2000.

[25] S. Todorcevic. A problem of von Neumann and Maharam about algebras supporting continuous submeasures. Fund. Math., 183:169-183, 2004. 